\title{
Religiöse Plakatwerbung: Ziele, Analyse, Kritik
}

\author{
von Jörg Scheibe
}

Mit Selbstverständlichkeit stellen heute konfessionelle Gruppen die Plakatwerbung in ihren Dienst. Die Plakate einzelner Organisationen ("Adveniat“, „Brot für die Welt") sind zu einem festen Begriff geworden. Kirchliche Veranstaltungen - von Gemeinde-Erntedankfesten bis zu Kirchentagen - werden durch Plakate angekündigt, mit unterschiedlichem Erfolg freilich. So nimmt die hier vorliegende Untersuchung einen aktuellen Anlaß auf. Es wurden zu dieser Untersuchung Gruppen und Institutionen, die Träger der Plakataktionen, befragt und so ein Teil der hier verwandten Daten gewonnen. Als Aufhänger sei ein neueres Beispiel angeführt:

Eines der letzten mit großem Aufwand verbreiteten Plakate war das zum 82. Deutschen Katholikentag in Essen 1968. Schlaglichtartig soll es hier das Dilemma religiöser Plakatwerbung verdeutlichen: Die DIN A 1-bis DIN A 3-formatigen Plakate (vgl. Abb. 1) führen den Text: „82. Deutscher Katholikentag 1968 Essen. 4.-8. September. Das Thema: ... mitten in dieser Welt. Heiße Probleme ... Offene Gespräche ... Moderne Formen der Begegnung ... Tage reichen Erlebens ... Glaubende ... Z Zweifelnde ... Suchende ... Wissende ... Mutige ... Angstliche ... Fordernde ..., viele Tausende kommen nach Essen - warum nicht auch Sie!"

Der graphische Bestandteil stellt eine weiße Spirale dar, die sich auf rotem Grund um einen durchkreuzten Kreis verengt und ein Drittel der Plakatfläche beansprucht. Das eigentliche Thema des Katholikentages: „Mitten in dieser Welt" ist kleingedruckt und nicht gesondert plaziert. Hauptzeilen bilden dagegen die Worte: „82. Deutscher Katholikentag 1968 Essen " im oberen Plakatteil und: "auch Sie!" im unteren Teil des Plakates. Dieses „auch Sie!“ büßt seinen - wohl beabsichtigten Appellcharakter zum einen dadurch ein, daß die Frage "warum nicht auch Sie?" mit einem Ausrufezeichen verundeutlicht wird. Zum anderen bilden die Hauptzeilen, die schon auf kurzer Distanz zusammengehörig erscheinen, keine verständliche Aussage.

Auf diesem Hintergrund wird das Ergebnis einer Befragung über dieses Plakat, die der Verfasser auf dem Katholikentag selbst durchführte, verständlich. Durchweg alle 30 Befragten gaben an, das Plakat zu kennen; bei näherer Befragung stellte sich jedoch heraus, daß sie sich an die Graphik nicht erinnerten oder nur den Bestandteil, der auch auf Programmen und Plaketten abgebildet war. Die auffälligere weiße Spirale kannte nur einer. Noch deutlicher wurde es beim Text: 50 v. H. glaubten, das Thema "mitten in dieser Welt" bilde allein den Text, obwohl diese Worte nur in einer Nebenzeile erscheinen. Vom gesamten übrigen Text, besonders den hervorgehobenen Worten "auch Sie!" war niemandem etwas in Erinnerung geblieben. Nur wenige sahen einen Zusammenhang zwischen Text und Graphik,

Dieser Beitrag stellt den Auszug einer Arbeit dar, die im Rahmen des von Dr. Gerhard E. Stoll geleiteten Seminars über "Religiöse Werbung“ im Wintersemester 1967/68 am Institut für Publizistik der Universität Münster entstand. Jörg Scheibe (Hannover) studiert an der Universität Münster Germanistik, Publizistik und Psychologie. 
niemand konnte ihn erklären. (Beispiele: „Christus ist der Eckstein ..." „Wir sind der Katholizismus..."). Es bleibt festzustellen, daß dieses Plakat seiner Aufgabe, eine Erstinformation zu bieten und die Veranstaltung $\mathrm{zu}$ "signalisieren“, nicht gerecht geworden ist.

Dieser jüngere Mißgriff auf dem Gebiet der religiösen Plakatwerbung wurde bewußt an den Anfang gestellt, da an diesem Beispiel typische Versäumnisse und Mängel deutlich werden, die auch bei anderen Erscheinungen häufig festgestellt werden konnten.

\section{Ziele der religiösen Plakatwerbung}

Religiöse Publizistik dient vorwiegend der Werbung. Uneinigkeit besteht jedoch über den Standort religiöser Werbung in der herkömmlichen Aufgliederung nach ideeller, kommerzieller und Vertrauenswerbung. Wenn es Aufgabe der Werbung ist, latente Bedürfnisse offenbar zu machen und ihnen eine Richtung zu geben, wird man das Werben um Vertrauen nicht als Werbeziel verstehen können; man muß es kommerzieller und ideeller Werbung beiordnen als Werbemethode. So ist religiöse Werbung eine Form ideeller Werbung, deren wesentlicher Bestandteil dann auch das Werben um Vertrauen ist. Als mögliche Ziele kirchlicher Plakatwerbung sind fünf thematische Gruppen denkbar, die jedoch in unterschiedlicher Ausprägung in Erscheinung treten ${ }^{1}$ :

Unterrichtung über Veranstaltungen, über die christliche Lehre. Bei näherer Untersuchung stellte sich heraus, daß sich die unterrichtenden Plakate auf die Ankündigung von Veranstaltungen im kirchlichen Raum beschränken, wobei das Plakat naturgemäß über die reine Ankündigung hinaus einen mehr oder weniger starken Appell vermittelt, die Veranstaltung auch zu besuchen.

Abbau von Vorurteilen gegen die Kirche, gegen die Geistlichen, gegen die Gläubigen. Es zeigte sich, daß das Feld der werbenden Selbstdarstellung völlig brachliegt.

Zeitgemäße Verkündigung der christlichen Lehre. Die Grundsätze und Glaubensinhalte der christlichen Lehre werden höchstens in Form von Bibelzitaten angeboten. So machen es sich vorwiegend die örtlichen Stadtmissionen zur Aufgabe, ausgewählte, unkommentierte Textstellen aus der Bibel - häufig auf Schriftplakaten - zu veröffentlichen. Der Ecclesia-Verlag („Bildpredigt") ließ 1961/62 die zehn Gebote mit Erläuterungen erscheinen. Allgemein beschränkt sich die Verkündigung auf das reine Schriftplakat.

Mission: Im Vordergrund steht die Volksmission oder Innere Mission. Einheitliche Thematik ist die Mahnung, die christliche Lehre auf das eigene Leben anzuwenden. Auf dieser Grundlage werden "Lebenshilfen" angeboten oder Forderungen an die christliche Nächstenliebe gestellt. Im einzelnen werden sehr konkrete Themen (z. B. Film- und Plakataktion: „25. Dezember - Lade einen Einsamen ein") aufgegriffen, doch ist der Bezug zu aktuellen Problemen (z. B. Bildpredigt: „Schlüsselkind Wirtschaftswunderkind") selten.

Spendenwerbung: Zunächst scheint die Spendenwerbung der Kirchen (z. B. „Misereor ${ }^{\alpha}$, Brot für die Welt“) einem ökonomischen Ziel zu dienen und damit in die Nähe der Wirtschaftswerbung zu rücken. Es darf jedoch nicht unbeachtet bleiben, 
daß in diesem Fall nicht für eine Ware, sondern für eine Sache: - äußere Mission, Dienst am Nächsten - geworben wird, deren Verwirklichung von der Opferbereitschaft der Umworbenen abhängt. Die Spendenwerbung ist deshalb durchaus zur kirchlichen Werbung als einer Form ideeller Werbung zu zählen, da sie eine Aufgabe der Kirchen, die Caritas, werbend unterstützt. Vorgefunden wurden folgende Formen der Spendenwerbung: a) äußere Mission; b) wirtschaftliche Unterstützung der Entwicklungsländer; c) spezielle kirchliche Anliegen (z. B. Unterstützung der Diaspora durch das Bonifatiuswerk).

\section{Zur Methode der Arbeit}

Der Untersuchung liegen die Angaben zugrunde, die auf dem Wege einer postalischen Fragebogenaktion ermittelt werden konnten. Ca. 200 Plakate waren zur Auswertung verfügbar. Weitgehend außer acht gelassen wurden reine Schriftplakate sowie Plakate ohne Bezug zu den Konfessionen. Den Trägern der Plakataktionen wurde folgender Fragenkatalog vorgelegt: 1. Handelt es sich um unregelmäßig erscheinende Einzel- oder Reihendrucke? - 2. Welche Auflage hatten die Plakate? - 3. Seit wann wird die Plakatwerbung durchgeführt? - 4. Wie hoch sind die Kosten einer Aktion? - 5. Auf welches (geographische) Gebiet erstreckt sich eine Aktion? Werden Schwerpunkte gebildet? - 6. Werden Meinungsumfragen durchgeführt? Wenn ja, mit welcher Fragestellung und welchem Ergebnis? - 7. Wurde die Werbewirksamkeit der Plakate getestet? - 8. Welche Graphiker wurden beschäftigt? - 9. Wurden die Plakataktionen gleichzeitig durch andere Werbemittel ergänzt? Welcher Anteil der Werbekosten entfiel dabei auf die Plakatwerbung? - 10. Wie kam der Plakattext zustande? - 11. Wird die Plakatwerbung zur Zeit ausgebaut oder zu Gunsten anderer Werbemittel eingeschränkt?

\section{Die befragten Institutionen}

\section{Evangelische Kirchentage}

Werbeziele: Werbung für die Veranstaltungen; Plakatwerbung seit: 1950; Verbreitung: Schwerpunkte sind der letzte Kirchentagsort, die gastgebende Landeskirche, der augenblickliche Kirchentagsort, Berlin und ausgewählte Hauptbahnhöfe im Bundesgebiet; Anschlagflächen: Litfaßsäulen, Werbeflächen, Schaukästen im Gemeindebereich; Kosten einer Aktion: 15000 DM; Graphiker: seit 1961 Hans-Jürgen Rau; Formate: DIN A 1, DIN A 0; Auflage: - -

S

\section{Bischöfliche Aktion "Adveniat"}

(Von der katholischen Kirche getragene Einrichtung für die Seelsorge in Lateinamerika). Werbeziele: Spendenwerbung für die äußere Mission; Plakatwerbung seit: 1961 (jeweils im Dezember ein Werbeplakat und eine Photoplakatserie); Verbreitung: BRD und Westberlin; Anschlagflächen: Litfaßsäulen, Schaukästen; Kosten einer Aktion: - - ; Graphiker: jeweils verschiedene; Formate: DIN A 2 (Auflage 80 000), DIN A 1 (Auflage 26 000), DIN A O (Auflage 23500 ). 


\section{Aktion „Brot für die Welt ${ }^{*}$}

(Von der evangelischen Kirche getragene Einrichtung für die kirchliche Entwicklungshilfe). Werbeziele: Spendenwerbung für die äußere Mission; Plakatwerbung seit: 1959; Verbreitung: BRD, Westberlin; Bildung von Schwerpunkten ist landeskirchlicher Initiative überlassen; Anschlagflächen: kirchliche Räume, Litfaßsäulen; Kosten einer Aktion: 25000 DM (jährlich für ein Standard- und ein Ergänzungsplakat); Graphiker: Rudi Wagner, Hans-Jürgen Rau; Formate: DIN A 3 DIN A 0; Auflage: Standardplakat $115000-142000$, Ergänzungsplakat 36000 bis 43000 .

\section{Evangelisch-katholische Film- und Plakataktion}

(Evangelisch-katholisches Unternehmen, das auf gemeinnütziger, nicht geschäftlicher Basis arbeitet). Werbeziele: Innere Mission; Plakatwerbung seit: 1964; Verbreitung: BRD, Osterreich, Schweiz; Anschlagflächen: Schaukästen, Warteräume, Betriebe, Litfaßsäulen, Plakattafeln; Kosten einer Aktion: - -; Graphiker: Gerhard Juchem; Formate: DIN A 6 bis $280 \mathrm{~cm} \times 200 \mathrm{~cm}$; Auflage: 12 000, Nachdruck bis zu 45000 .

\section{Männerarbeit der evangelischen und katholischen Kirche}

Werbeziele: Innere Mission; Plakatwerbung seit: 1958; Verbreitung: BRD, zuweilen lokale Schwerpunkte; Anschlagflächen: sämtliche öffentlichen und kirchlichen Anschlagmöglichkeiten; Kosten einer Aktion: 2000 bis 3000 DM; Graphiker: nicht hauptamtlich beschäftigt; Formate: DIN A 3 und DIN A 2; Auflage: 12000 bis 20000.

\section{Ecclesia-Verlag (Bildpredigt)}

Werbeziele: Innere Mission; Plakatwerbung seit: 1947 (Wiesbaden); Format: DIN A 2; (Weitere Informationen lagen nicht vor).

\section{Bonifatiuswerk}

Werbeziele: Werbung für die Diasporaarbeit; Plakatwerbung seit: 1917; Verbreitung: BRD; Anschlagflächen: Schaukästen im Gemeindebereich; Kosten: - -; Graphiker: wird nicht beschäftigt; Auflage: 25000 bis 30000 .

\section{Päpstliches Werk der Glaubensverbreitung}

(Kirchenamtliche Institution, die unmittelbar der "Congregatio Propaganda Fide" in Rom untersteht). Werbeziele: Außere Mission; Plakatwerbung seit: 1931 (jährlich ein Plakat); Verbreitung: alle katholischen Pfarreien der BRD mit Ausnahme von Bayern; Anschlagflächen: Gemeindebereich; Kosten: - - ; Graphiker: nur gelegentlich beschäfrigt; Auflage: 30000 .

\section{Bibelanstalt Stuttgart}

Werbeziele: Absatzwerbung für die Stuttgarter Bibelausgabe; Formate: DIN A 2, DIN A 3; (Weitere Informationen lagen nicht vor). 


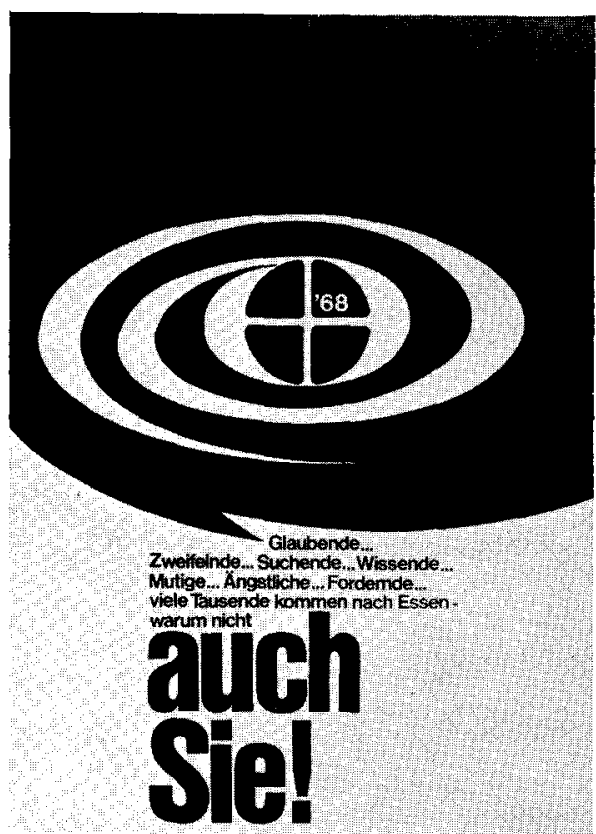

Abb. 1, Seitc 235: Katholikentag Essen 1968

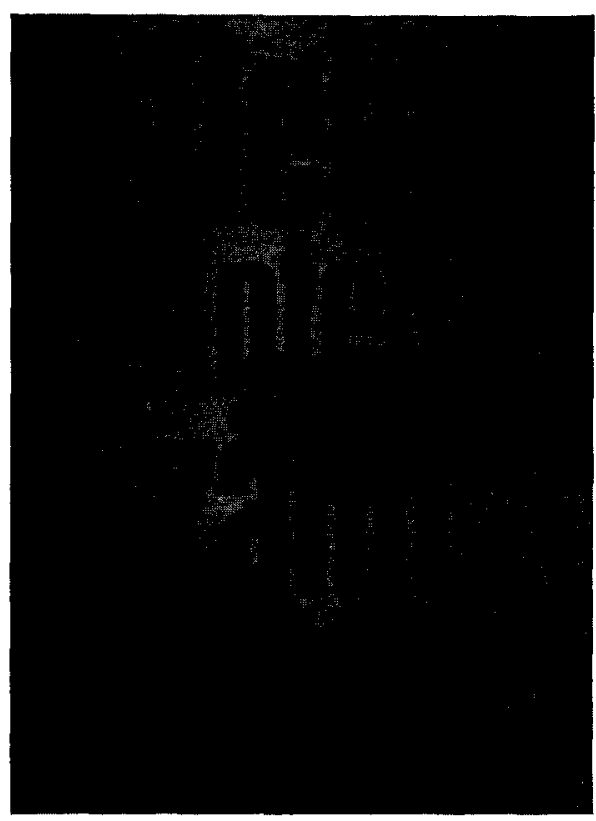

Abb. 3, Seite 239: Aktion Adveniat

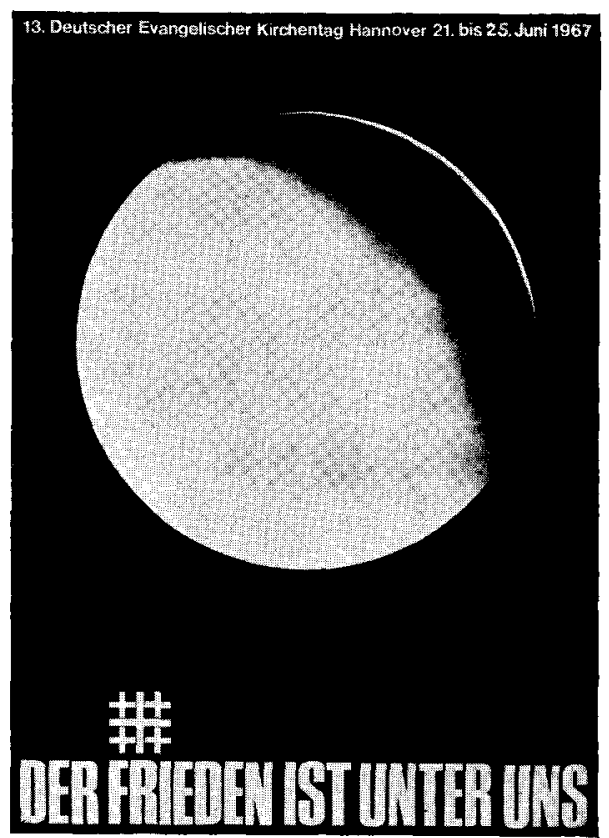

Abb. 2, Seite 239: Evangelische Kirchentage

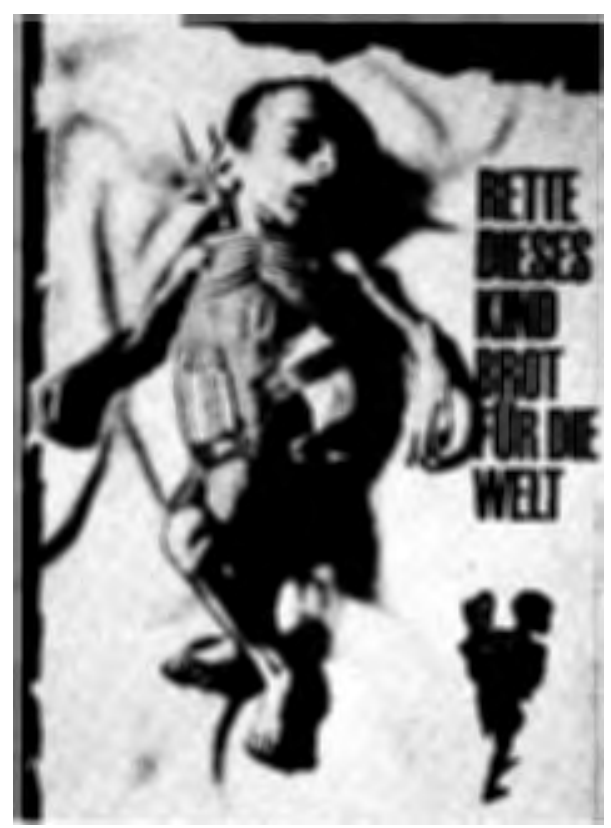

Abb. 4, Seite 239: Brot für die Welt 
Abb. 5, Seite 239: Film- und Plakataktion

Abb. 6, Seite 240: Männerarbeit der Evangelischen Kirche
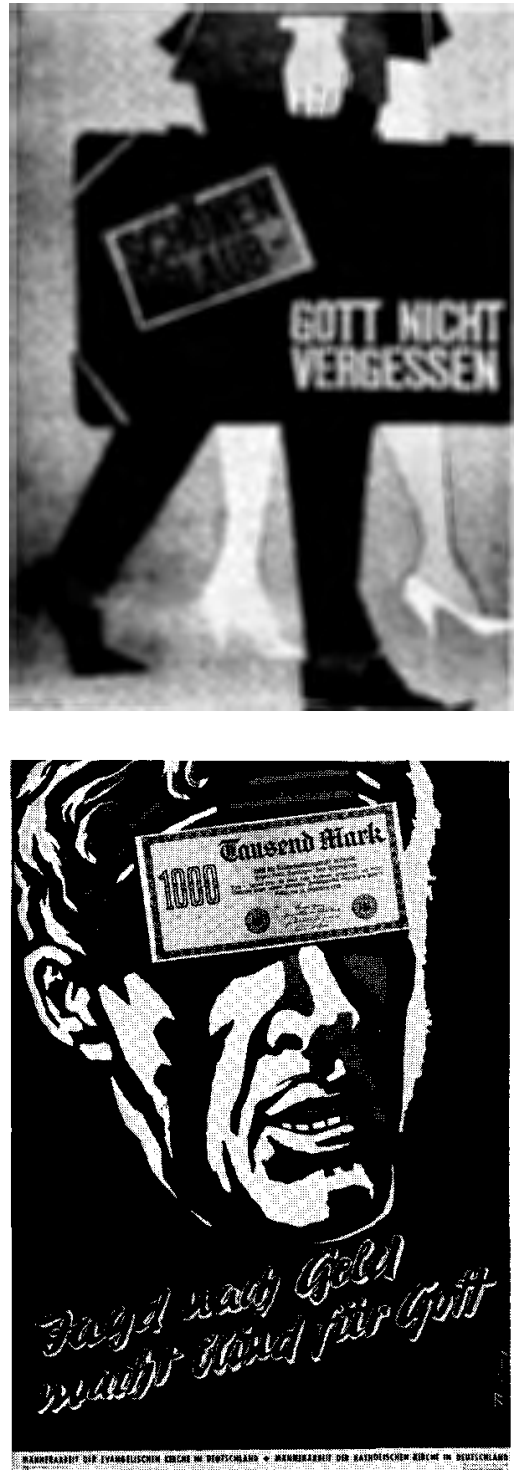

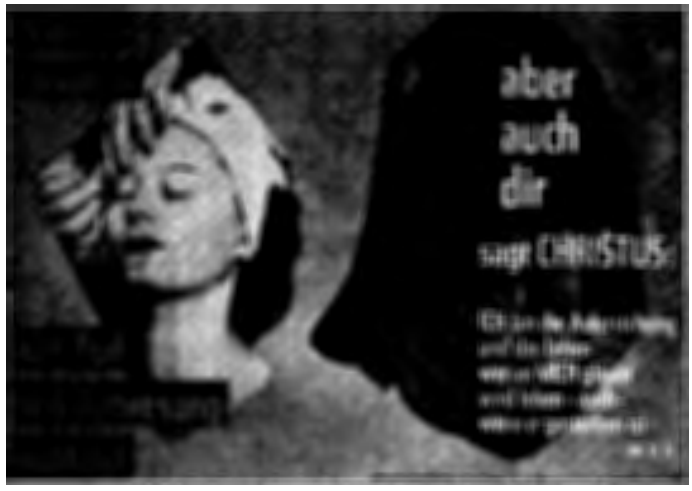

Abb. 7, Seite 240: Bildpredigt / Ecclesia-Verlag

Abb. 8, Seite 240: Bonifatius-Werk

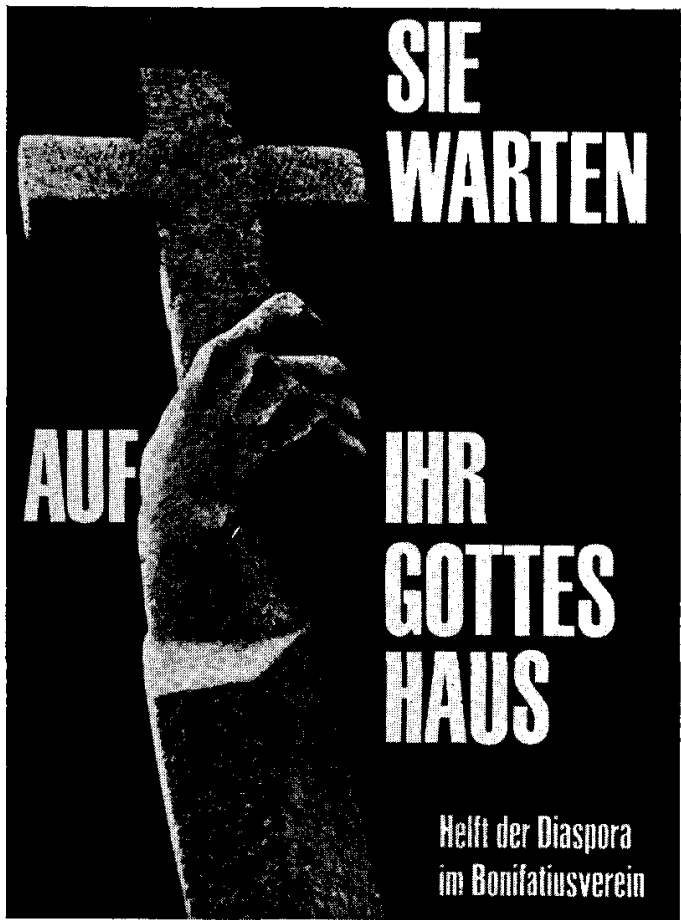




\section{Berliner Stadtmission}

Werbeziele: Innere Mission, Ankündigungen und Werbung für Veranstaltungen; Verbreitung: Westberlin, auch BRD; Anschlagflächen: sämtliche öffentlichen und kirchlichen Anschlagmöglichkeiten; Graphiker: jeweils wechselnd; Formate: DIN A 2, DIN A 1.

\section{Weitere Stadtmissionen}

(Die Stadtmissionen, in ihrem örtlichen Bereich selbständig arbeitend, sind vorwiegend zusammengefaßt in der "Arbeitsgemeinschaft Christlicher Plakatmissionen", Stuttgart. Ihre Plakate entstehen durch eigene Entwürfe, Zusammenarbeit mit anderen Plakatmissionen oder Austausch. Nähere Angaben lagen von den Stadtmissionen Hannover, Hamburg und Wuppertal vor.) Werbeziele: Innere Mission, Ankündigung von und Werbung für Veranstaltungen; Verbreitung: lokal begrenzt; Anschlagflächen: örtliche Verkehrsmittel, Schaukästen, Litfaßsäulen; Kosten einer Aktion: ca. 500 DM; Graphiker: werden selten herangezogen; Formate: DIN A 4 bis DIN A 1; Auflage: um 500.

\section{Analyse der nach 1945 erschienenen Plakate}

Im folgenden sollen die Plakate der einzelnen Institutionen in Bezug auf graphische Gestaltung, Text, Komposition, Farbgebung, Verständlichkeit und Blickfangwirkung stichwortartig analysiert werden.

Plakate der evangelischen Kirchentage: Wiederkehrendes Motiv: fünf symmetrisch angeordnete Kreuze (Kirchentagssymbol), Erinnerungswert! Großflächige Graphiken - z. T. mit Photoelementen -- dienen dem Blickfang. Text: Information über Ort und Zeit der Veranstaltung; Thena des Kirchentages (drei bis fünf Worte). Knapper Text, einfache graphische Form und großes Format bewirken gute Verständlichkeit. Thema und Graphik sind gut verbunden. (Vgl. Abb. 2).

Plakate der ${ }_{\text {Aktion Adveniat }}$ : Starker Appellcharakter durch Skizzenhaftigkeit der Graphiken, Hervorhabung des Possessivpronomens und die Gebärde des Bittens. Text: wie die Bildelemente schwarz gedruckt, erstreckt sich über die halbe Plakatfläche, fünf bis sechs Worte. Einheitlicher Gesamteindruck, unmittelbar verständliche Aussage durch knappc, einfache Gestaltung. (Vgl. Abb. 3).

Plakate der Aktion „Brot für die Welt": Standardplakat („Hungerhand“): einfache, in der Komposition ausgewogene Formen. Eine braune Hand reckt sich nach dem weiß gedruckten Wort "Brot", das dadurch zum Symbol wird und bewußt als graphisches Mittel eingesetzt ist. Eindringlicher Appellcharakter. Ergänzungsplakate: Gleicher Text, Interpretation durch andere Bildelemente. Appell an das Emotionale. Außerste Beschränkung im Text, besonderer Gedächtniswert durch gleichbleibende Aussage. (Vgl. Abb. 4).

Plakate der Film- und Plakataktion: Auffällige graphische Mittel, Mehrfarbendruck, guter Bezug zwischen Text und Graphik. Themen: sozial-ethische Probleme unter christlichem Aspekt. Text: fünf bis sechs Worte, längerer Text wird in Haupt- und Nebenzeilen gegliedert. Aussage verständlich, schlechte „Fernwirkung" durch zu kleine Formate. (Vgl. Abb. 5). 
Plakate der „Männerarbeit": Häufig dunkler Hintergrund mit kontrastierender verschiedenfarbiger Schrift. Unruhige Momente in der Graphik, unregelmäßig gesetzte Schrift und schlagwortartiger Text dienen dem Blickfang. Neben Stellungnahmen zu aktuellen ethischen Problemen überwiegen speziell christliche Themen. Unterschiedliche Gestaltung: die Texte wirken z. T. sloganartig; oberflächlicher Bezug zu den mehr illustrierenden Bildelementen. Formate zu klein gewählt. (Vgl. Abb. 6).

Plakate des Ecclesia-Verlags ${ }^{2}$ : Vorwiegend Photomontagen, durch die bestimmte menschliche Situationen dargestellt werden. Farben werden häufig ohne Bezug auf das - in Grauwerten erscheinende - Bildelement eingesetzt (Ausfüllung leerer Flächen, Schriftgrund). Text: 20 bis 50 Worte (!), unübersichtlich angeordnet, vordergründiger Zusammenhang mit der Graphik, häufig Bibelzitate oder Formulierungen in sehr einfachem Sprachstil: z. B. „Schluß mit den dreckigen Filmen im heuchlerischen Gewand der Kunst“. Text, Graphik, Komposition und Format sind kaum geeignet, einen Betrachter über größere Distanz anzusprechen. (Vgl. Abb. 7).

Plakate des Bonifatiuswerks: Wiederkehrende Verwendung des Kreuzsymbols und der Aufforderung „Helft der Diaspora“. Relativ kurzer Text, Blickfangwirkung. Bei dem Betrachter, der nicht über die Arbeit dieser Einrichtung informiert ist, wird jedoch kaum ein unmittelbares Interesse an der Plakataussage geweckt werden.

Plakate des Päpstlichen Werks der Glaubensverbreitung: Text häufig über das gesamte Plakat gedruckt, selten bewußt plaziert. Die Graphiken und Photomontagen gewinnen selten einen eigenen Aussagewert. Allgemeine, etwas flache Begründung des Anliegens („Die Völker brauchen Christus“). Unübersichtliches Gesamtbild. Vermutlich geringe Wirkung auf Außenstehende. (Vgl. Abb. 8).

Plakate der Bibelanstalt Stuttgart: Einheitliches Motiv ist die Bibel. Der Text erinnert an moderne Slogans der Wirtschaftswerbung. Graphik und Komposition muten wenig durchdacht an. Die im Superlativ formulierte Aussage wird auf den Betrachter nur eine geringe Wirkung ausüben. Formate sehr klein!

Plakate der Berliner Stadtmission: Stark stilisierte Graphiken mit Signalcharakter, sparsame Verwendung von Farben, besonders "plakative" Texte. Ausgewogene Komposition von Schrift und Bild, Reduzierung der graphischen Formen auf ein Mindestmaß.

Weitere Stadtmissionen: Text: Ủberwiegend Wiedergabe von Bibelzitaten. Spärliche Verwendung graphischer Elemente. Die eigenen Entwürfe einzelner Stadtmissionen sind auf Grund des oft winzigen Formats und der ausführlichen Texte nicht geeignet, den Betrachter zu fesseln und die Aussage verständlich werden zu lassen. Gemeinschaftsproduktionen vermögen zuweilen graphisch und kompositorisch besondere Wirkung zu erzielen.

\section{Kritik}

Das Plakat, das seine Werbebotschaft dem Empfänger optimal (schnell und nachhaltig) übermitteln soll, muß sich heute mehr und mehr um eine Verdichtung der graphischen und sprachlichen Aussage bemühen. Diese Reduktion des Inhalts darf dabei jedoch nicht auf Kosten der Verständlichkeit gehen. Wenn der Kommunikator dem Werbemedium "Plakat“, dessen Wirkungsweise sich von anderen Werbemedien vielfältig unterscheidet, in der Gestaltung - besonders in der Komposition - 
gerechtwerden will, ist es erforderlich, daß ausnahmslos geschulte Fachkräfte, Graphiker, Psychologen und Werbefachleute, mit der Herstellung beauftragt werden. Es ist zu untersuchen, inwieweit die genannten Voraussetzungen in der religiösen Plakatwerbung anerkannt bzw. erfüllt werden.

Die graphiscbe Gestaltung der Plakate obliegt bei sieben von elf befragten Institutionen ausgebildeten Fachleuten. Bei diesen Einrichtungen hat das zur Folge, daß zwei Grundregeln der Plakatgestaltung weitgehend erfüllt sind: einprägsame, „signalisierende “ Graphik, kurzer, schlagwortartiger Text. Bei den übrigen (Bonifatiuswerk, Päpstliches Missionswerk, Stadtmissionen) wird entweder nie oder nur gelegentlich ein Graphiker hinzugezogen. Auffällig ist hier dementsprechend die meist überladene, dilettantische Aufmachung. Plakate, deren Graphik nur in einem vordergründigen Bezug zum eigentlichen Thema steht, indem sie sich auf die reine Illustration beschränkt, sind weitaus in der Uberzahl. Nur selten erlangt die Graphik eine eigene Aussagekraft wie z. B. in dem Grundmotiv des Plakats „Brot für die Welt" oder den Plakaten der Berliner Stadtmission. Generell bestätigt sich, daß dem Layout der Plakate bei steigenden Auflagen und Aufwendungen entsprechend mehr Sorgfalt zugewandt wird.

Ein beträchtlicher Teil der Plakattexte wird entweder wörtlich oder in abgewan delter Form aus der Bibel übernommen. Moderne Übersetzungen haben das LutherDeutsch verdrängt. Die Stadtmissionen, die fast ausschließlich Bibelzitate verwenden. gehen davon aus, daß das - ursprünglich situationsgebundene - Bjbelwort auch für den heutigen Menschen und zu aktuellen politischen oder sozialen Problemen Geltung hat und deshalb auch als Plakattext geeignet ist. Hier liegt ein grundlegender Irrtum vor. Ein gelungener Plakattext muß mehrere Voraussetzungen erfüllen: er muß von bildhafter, einprägsamer Knappheit sein und zugleich einen ausgewogenen Rhythmus besitzen. Das bedeutet, daß jeder Text bewußt für das Plakat geformt werden muß und nicht ohne weiteres aus vorhandenen Quellen übernommen werden kann. Die Tatsache, daß die Bibel die Grundlage christlichen Glaubens ist, bietet keine Gewähr dafür, daß die einzelnen Verse daraus auch gute Plakattexte abgeben. Wenn manche Bibelzitate geeignet sind, wie z. B. der einprägsame, rhythmisch akzentuierte Spruch „Gott ist Liebe", so ist das Zufall und darf nicht dazu verführen, der Einfachheit halber Bibelzitate grundsätzlich für wirksam zu halten. So ist die Veröffentlichung der Hamburger Plakatmission: „Als die Zeit erfüllt war, sandte Gott seinen Sohn (Galater 4,4$)^{\star}$ völlig ungeeignet, da der Text weder „plakativ" ist, noch das Anliegen klar ersichtlich wird. Hinzu kommt, daß Bibeltexte häufig in Verschlüsselungen oder Bildern sprechen, so daß der Betrachter zuerst die Aussage verstehen und dann auf seine Situation oder Zeit beziehen muß. Doch auch wenn ein freier Text verwendet wird, finden sich holprige und klanglose Satzgebilde wie: „Ihr feiert Weihnachten, das Fest Christi Geburt, aber wer von euch kennt Ihn denn?“ (Bildpredigt Nr. 316).

Die entscheidende Schwäche bei der Gestaltung ist jedoch in der Länge der Texte zu suchen. Schon die Tatsache, daß die - in der kommerziellen Werbung in dieser Form unüblichen - „Textplakate" noch immer weit verbreitet sind, läßt auf eine gewisse Kurzsichtigkeit der Hersteller schließen. Ein „Schnelldialog“", der nur etwa sechs Sekunden dauern soll, ${ }^{3}$ ist bei einer Wortzahl von zehn bis 50 völlig ausgeschlossen. Die Herausgeber führen zum Teil als Begründung an, daß die Textplakate nur dort verwandt werden, wo der Betrachter längere Zeit verweilen wird. Offensichtlich besteht hier die Meinung, daß der Betrachter ein religiöses Plakat mit langem Text bis zum Schluß durchlesen wird, wenn er nur genügend Zeit dazu hat. 
Die Herausgeber haben jedoch nie überprüft, ob nicht auch in diesen Fällen ein Plakat, das nach den Gesichtspunkten des "Signalplakats" gestaltet ist, bessere Dienste leistet. Ein Mehr an Text muß in der Plakatwerbung nicht ein Mehr an Aussage- und Überzeugungskraft bedeuten. Hinzu kommt, daß der Grundsatz, Textplakate zumindest nicht an großen öffentlichen Werbeträgern auszuhängen, häufig nicht beachtet wird.

Die konstatierten Schwächen in der Gestaltung einiger Plakate lassen sich zurückführen auf schwerwiegendere Versäumnisse, die auf dem Gebiet der Motivforschung und der Planung liegen. Bezeichnend für die Einstellung zum Umworbenen ist die Tatsache, daß von den untersuchten Einrichtungen allein die evangelisch-katholische Film- und Plakataktion vor der Veröffentlichung eines Plakats Meinungsumfragen anstellt. Die Plakate der Männerarbeit der evangelischen und katholischen Kirche werden nach dem Erscheinen auf ihre Werbewirksamkeit getestet. Andere Institutionen leiten die positive oder negative Aufnahme ihrer Plakate nur aus Bestellungen oder Zuschriften ab. Anscheinend ist den meisten Herausgebern die Notwendigkeit einer Erforschung der Bewußtseins- und Bedürfnisstruktur der zu Umwerbenden nicht bewußt oder sie gehen noch immer von der Vorstellung aus, daß ihre Werbebotschaft sich an eine "gefestigte Gemeinschaft Gleichgesinnter" richte. Vielleicht spielt bei diesem Versäumnis auch eine - unbewußte - Besorgnis mit, eine solche Motivations- und Wirkungsforschung könne eventuell desillusionierende Aufschlüsse zeitigen. Doch gerade, weil auf dem Gebiet religiöser Werbung ein „Erfolg“ nicht ohne weiteres ablesbar ist und auch ein guter Graphiker nicht ermessen kann, welche Assoziationen oder Emotionen - erwünscht oder unerwünscht - sein Plakat hervorrufen wird, ist eine "Strategie im Reich der Wünsche" gerade auch für die Kirchen und ihre Werbebemühungen unerläßlich.

Dringend notwendig wäre eine Nachprüfung, ob die persönliche Ansprache im $\mathrm{Pla}$ kattext stets das "Du“ verwenden sollte, wie es in der konfessionellen Plakarwerbung generell der Fall ist, oder welche Wirkung Bibeltexte auf Außenstehende ausüben. In Bezug auf die Themenauswahl reicht es nicht, wenn die Kirchen nur von ihrem missionarischen Auftrag ausgehen. Bevor sie den Menschen durch ihre Werbung ermahnen, erziehen oder bekeliren, müssen sie ihn sehen, wie er ist, nicht, wie sie ihn gern hätten. Unerläßlich ist ebenfalls eine Wirkungskontrolle im Anschluß an eine Plakataktion, um daraus Erkenntnisse für die Planung zu gewinnen. Publizistisch gesehen heißt das, daß die Kommunikation durch das Plakat nicht länger ein Einbahnweg vom Kommunikator zum Rezipienten bleiben darf, sondern zu einem Dialog ausgebaut werden muß. Mit der Indienstnahme dieses Publikationsmediums ist zwangsläufig die Übernahme der spezifischen Handhabung verbunden.

Bemüht, sich in Bezug auf Gestaltung, Plazierung und Appellwirkung von der Konsumwerbung abzuheben, ist die religiöse Plakatwerbung viele Wege gegangen („Textplakate", Bibeltexte, spezielle Anschlagflächen etc.), doch bei dem Bemühen um Distanzierung wurden häufig auch selbstverständliche Grundregeln und technische Hilfsmittel außer acht gelassen oder abgelehnt. Außer bei den großen Institutionen, die Spendenwerbung betreiben, oder bei den Kirchentagen findet sich keine durchgehende Planung für die Plakatwerbung. Die genannten Einrichtungen müssen sich am Erfolg ihrer Werbung orientieren und dementsprechend planen. Bei den übrigen finden sich immer wieder folgende Versäumnisse und Mängel:

Man verzichtet auf eine jeweils gezielte Streuung und veröffentlicht immer nach demselben "Verteiler". Viele Aktionen werden einmalig, ohne Unterstützung durch 
andere Werbemittel durchgeführt und begeben sich so der intensivierenden Wirkung der Wiederholung und Ergänzung. Die Formate sind bis auf sehr wenige Ausnahmen zu klein gewählt. Während die kommerzielle Werbung schon ganze Werbeflächen (16 Bogen und mehr) für ein Motiv ausnutzt, beschränken sich die Herausgeber religiöser Plakate auf DIN A 1- bis DIN A 4-Formate. Bei manchen Institutionen (z. B. Bonifatiuswerk) besteht noch eine unverständliche Scheu vor den öffentlichen Anschlagflächen, die wohl ebenfalls daher rührt, daß man eine Distanzierung von der Konsumwerbung anstrebt. Kirchenschaukästen und - meist sehr dunkle Kirchenvorräume als Anschlagorte erschweren die Verbreitung der Werbebotschaft auf Außenstehende. Voraussetzung für eine Ausdehnung des Wirkungsradius ist auch hier eine zweckentsprechende "plakative" Gestaltung.

Erfordert die Aufmachung vieler Plakate schon eine notwendige Beschränkung in der Wahl des Ausstellungsortes, so schränkt die Thematik häufig noch den Kreis der Anzusprechenden ein. Plakattexte mit Missionscharakter begründen ihren Appell mit der christlichen Lehre oder veröffentlichen Textstellen aus der Bibel und setzen damit voraus, daß der Betrachter auf dem Boden dieser Lehre steht. Hier wäre zu fragen, ob es nicht Aufgabe religiöser Werbung sein sollte, verstärkt diejenigen anzusprechen, die diesen Boden schon verlassen haben oder ihn zu verlassen im Begriff sind. Dabei darf es dann nicht mehr genügen, daß den Fragen und $Z$ weifeln der Menschen mit den Geboten („Bildpredigt“ Nr. 294: „6. Gebot: Du sollst nicht ehebrechen. Vergiß nicht, daß schon beim harmlosen Wochenendflirt der Ehebruch beginnt!") oder christlich gefärbten Allgemeinplätzen ("Männerarbeit": „Gott sieht hinter Deine Maske") begegnet wird. Ein Ansatz ist die Arbeit der evangelischkatholischen Plakataktion, die an Hand ganz konkreter Alltagsprobleme Hilfen zu geben versucht. Es fällt auf, $d a ß$ in der Thematik ein möglicher Bereich religiöser Werbung durchweg ausgespart bleibt: die sogenannte Image-Pflege, deren Aufgabe es wäre, Vorurteile gegen die Kirche als Institution, gegen die Geistlichen und schließlich auch gegen die Gläubigen abzubauen. Erst auf der Grundlage gegenseitigen Verständnisses können die Werbebotschaften erfolgversprechend sein. Nach Meinung des Verfassers wäre gerade die Plakatwerbung geeignet, ein solches Verständnis zu fördern.

Ein einheitlicher sprachlicher wie formaler Stil kann den konfessionellen Plakaten nicht bescheinigt werden. Die Ausprägungen gehen vom unkommentierten "frommen" Bibelvers über bunte, überladene Sloganwerbung bis zu den künstlerisch und inhaltlich beispielhaften Plakaten etwa der evangelischen Kirchentage oder der Berliner Stadtmission. Es wäre ein falsches Bemühen, wollten die Herausgeber auf Kosten der Eigengesetzlichkeit des Plakats als Werbemedium einen besonderen "kirchlichen Stil" schaffen. Es scheint bei der augenblicklichen Situation kirchlicher Plakatwerbung viel angemessener, sich die Erfahrungen und Methoden der Konsumwerbung unter Berücksichtigung der anders gelagerten Werbeziele zu Nutze zu machen. Das Anliegen und der selbst gestellte Auftrag sollten eine ausreichende Abgrenzung zur übrigen Werbung ermöglichen.

\section{Anmerkungen:}

1. Manfred Schütte gibt in seinem Buch „Kirchliche Werbung", Düsseldorf/Wien 1966, S. 83 eine ausführliche Darstellung der möglichen Aufgaben kirchlicher Werbung.

2. Von den 437 erschienenen Nummern lagen 93 Exemplare aus den Jahren 1960-1968 vor.

3. Vergl. Henk Prakke, Bild und Plakat, Assen, 1963, S. 30. 


\section{S U M M A R Y}

Poster advertising by the churches is studied with a sample of ca. 200 posters that were used by the catholic and protestant churches promoting conferences, missionary work and welfare activities. Analysis shows the church posters lacking advertising know-how. Some proposals are included for using communication research findings.

\section{RESUMEN}

Por el ejemplo de los carteles se examina la utilización de medios de propaganda moderna al servicio de las Iglesias. Para el análisis se disponía de alrededor doscientos carteles. Se hizo una encuesta entre algunas instituciones que suelen usar esta clase de propaganda por carteles, investigando acerca de datos de producción, gastos generales, tiraje, circulación y frecuencia. Entre las mencionadas instituciones se cuentan por ejemplo: el Congreso de la Iglesia Evangélica Alemana, las acciones „Adveniat" y „Pan para el mundo“, las misiones urbanas. El análisis crítico de los carteles usados por las Iglesias reveló una cierta insuficiencia e ineficacia debidas a una falta de criterio, especialmente respecto investigación de motivos y planificación. Para remediar esta situación se propone aprovechar las ciencias periodísticas y las experiencias hechas por los economistas. 